\title{
Magnetic field generated by the Weibel instability
}

\section{Chang-Mo Ryu*}

Pohang University of Science and Technology

E-mail: ryu201@postech.ac.kr

The origin of the magnetic field in the universe is of great interest. Biermann's battery and the Weibel instability have been considered as candidates for such mechanisms [1]. The Weibel instability is believed to play an important role in the Super Nova remnant (SNR) and in the inertial fusion plasma. In this paper, we discuss the evolution of current structures and magnetic fields of the nonlinear Weibel instability up to the saturation regime, based on PIC simulation results.

References

[1] D. Ryu, D. Schleicher, R. Treumann, C. Tsagas, and L. Widrow, Magnetic fields in the largescale structure of the universe, Space Science Reviews, 158, 35 (2011).

Frontiers of Fundamental Physics 14 - FFP14,

15-18 July 2014

Aix Marseille University (AMU) Saint-Charles Campus, Marseille

${ }^{*}$ Speaker. 